\title{
Distance discrimination and observer strategy in judgments of relative size'
}

\author{
JOHN A. WILLIAMSEN ${ }^{2}$ \\ UNIVERSITY OF ILLINOIS
}

Six paid Ss participated in an experiment designed to assess the effects of observer strategy and the detectability of a distance cue on judgments of relative size. Ss viewed stimulus pairs through a 21-in. tunnel at one end of an 8-ft table. The standard stimulus, $94 \mathrm{in}$. from $S$, was individually paired with three larger and two smaller comparison stimuli 96 in. from $S$. A slit of light on the table $95 \mathrm{in}$. from $S$ and 90 deg to his line of sight served as distance cue. Increases in distance detection improved accuracy of relative size judgments when the comparison stimulus was larger than the standard stimulus, but decreased accuracy when the comparison stimulus was smaller. Magnitude of this effect varied directly with $S_{s}$ 'postexperimental reports of judgmental strategy. These findings confirm the importance of distance discrimination in judgments of actual size and the necessity to control perceiver characteristics in the study of size-distance relationships.

It is generally agreed that judgments of the physical size of objects depend in some way on the perception of the distance of the objects from the O (Hastorf \& Way, 1952; Holway \& Boring, 1941; Over, 1960, 1963). Simplistic theories attempting to explain the relationship between perceived size and distance, such as the size-distance invariance hypothesis (Epstein, Park, \& Casey, 1961; Kilpatrick \& Ittelson, 1953), do not have general validity (Epstein, 1961, 1963; Gogel, Wist, \& Harker, 1963; Gruber, 1954; Jenkin \& Hyman, 1959). Attempts to generate more complex theories relating size and distance (Gilinsky, 1951, 1955b) have not met with any more success (Gruber, 1956; Smith, 1952), largely because they have been unable to account for the wide variety of empirical data on size-distance relationships obtained over the last twenty years.

The lack of a simple relationship between stimuli and responses in size-distance perception has led researchers to give renewed attention to $O$ variables, especially those factors variously labeled "attitude," "set," "strategy," etc. The effect of $S$ characteristics can be seen, for example, in "overconstancy," the tendency for the perceived size of an object to increase with physical distance (Gilinsky, 1955a; Jenkin, 1957; Smith, 1953). Carlson (1960) suggests that this tendency to overestimate the size of a farther stimulus when compared to a nearer object is the result of an overcompensation for differences in distance. He further suggests (Carlson, 1962) that the effects of $\mathrm{O}$ variables probably leads to biases in the judgmental response rather than the perceptual process itself. Epstein (1963) has replicated Carlson's (1962) study and reached similar conclusions regarding the importance of attitude in overestimation.

The present experiment investigates the effects of distance detectability on judgments of actual size. While previous studies have indicated in a general way that the presence of distance cues is necessary for judgments of actual size, they have been'so designed that they merely demonstrated an improvement in accuracy when normal viewing conditions were present over the case when all cues to distance were removed, and/or they have failed to obtain measurements of the detectability of the distance cues.

In the experiment reported here a single distance cue was varied in intensity and measurements of its detectability obtained for six Os. These Os also made judgments of the actual size of five comparison stimuli relative to a single standard stimulus at various distance cue detectability levels. This design made it possible to relate discrimination of actual size to the detection of distance. The results were expected to permit a more detailed answer to the question of the relationship between discrimination of actual size and distance detection and provide more information about the role of $\mathrm{O}$ attitude in size-distance judgments.

\section{Subjects}

\section{METHOD}

Six paid Ss, three males and three females, served as Os. The three males were graduate students at the University of Illinois. The females were wives of graduate students. All Ss had normal or fully-corrected vision.

\section{Stimulus Materials and Apparatus}

The comparison stimuli were five white posterboard squares, varying in size but otherwise identical. The standard stimulus (always placed 94 in. from $\mathrm{S}$ ) was $3-8 / 16 \mathrm{in}$. on a side and subtended a retinal angle of $2 \mathrm{deg} 8 \mathrm{~min}$. The five comparison squares were placed $96 \mathrm{in}$. from $\mathrm{S}$ and individually paired with the nearer square. These stimuli were $3-4 / 16$, $3-6 / 16,3-10 / 16,3-12 / 16$, and $3-14 / 16$ in., respectively. The sizes were selected on the basis of their relative retinal angle difference with the nearer square; these differences were, in order, $-11,-7,+4,+7$, and +11 min of angle.

The apparatus consisted of a table $4 \times 8 \mathrm{ft}$ with a 21 -in. viewing tunnel at one end for monocular viewing. A large screen placed in front of S's end of the table prevented him from seeing either the table or the room. In addition, a shutter in the tunnel remained closed between trials, preventing $S$ from viewing the table surface or stimuli. Head movement was restricted by a rubber face mask and a restraining board that rested on the S's head. The distal viewing aperture of the tunnel was $2 \times 2$ in.

The table was painted a dull black, with a similarly black background at its far end. Its surface was illuminated by a 40-W fluorescent bulb, appropriately shielded to reduce the amount of light in the experimental room. A slit, $30 \times 1 / 16$ in., was cut into the table 95 in. from S. This slit was undetectable under normal viewing conditions. However, when illuminated by two 150-W floodlamps housed in a lightproof box directly underneath the slit, it appeared as a lighted line perpendicular to S's line of sight. The floodlamps were connected to a Variac so that illumination could be varied.

\section{Procedure}

The first phase of the experiment was devoted to a determination of three Variac settings that would yield three discriminably different performances in distance detection for each individual $S$. These three settings, plus a "Variac-off" (no-cue) condition, were used in the size judgment trials which followed. Each of the five pairs of stimuli was presented at the four levels of distance discrimination, making it possible to relate detection of relative size to detection of distance. Idiosyncratic Variac settings for each individual $O$ were obtained in an attempt to induce comparable levels of distance discrimination among the Ss. At the beginning of the first session the following instructions were read to each $S$ as he followed on his copy: 
"In this experiment you will be shown several different pairs of squares. The two members of each pair will be presented one after the other and you are to tell me whether you think the second square is physically smaller or physically larger than the first. By physically larger (or smaller) I mean larger (or smaller) as measured by a ruler.

"Here is how the procedure works. When I say, 'Ready, one,' press the button and you will see the first member of the pair for two seconds. After a two second delay I will say, 'Ready, two.' Pressing the button again will expose the second member of the pair for two seconds. After you see the second square tell me if you believe it to be physically larger or smaller than the first. This will not always be easy, so don't be dismayed if you find the task a little difficult.

"On any given trial, the probability that the second square is larger (or smaller) than the first is $50 / 50$ or one out of two. If you are uncertain about your judgment, indicate this by saying 'one' after you make your size judgment. Thus, if you believed the second member of a given pair was larger than the first, but felt uncertain about your judgment, you would say 'Larger, one.' If you are moderately certain of your judgment, say 'two.' If you are quite certain that you are correct, say 'three.'

"You can always feel at least 50\% certain about your judgments, since by chance alone, you can be correct $50 \%$ of the time. I would like you to restrict your use of the confidence rating 'one' to those times when you are 50-65\% certain. Use 'two' when you are $65-80 \%$ certain, and 'three' when you are $80-100 \%$ certain. Note that you do not have to be completely uncertain to use a 'one' nor absolutely certain to use category 'three.' I will be keeping track of your responses during the practice trials until you have had sufficient practice using the scale. It is not really quite as complicated as it sounds.

"Do you have any questions?

"Here is an additional bit of information about the pairs of stimuli you will see. The individual members of any pair will never be at the same distance from you. That is, one of the pair members will always be farther than the other. To help you decide which stimulus is closer and which is farther from you, a line of light will appear from time to time running at right angles to your field of vision. If you see a square in front of the line you will know it is closer to you than the one you perceive to be behind the line. Thus the line will help you to detect the relative distances of stimuli making up a pair. This information should be of help to you in making your decision about which square is larger. When you view a pair of squares, one member of the pair will be in front of the line and the other member will be behind it. The probability that the second square will be behind the line is one out of two. However, a square that is behind the line will not always be larger (or smaller) than its pairmate in front.

"Do you have any questions now?"

Questions were answered by paraphrasing relevant sections of the above instructions.

Following the reading of the instructions, $\mathrm{S}$ began judging relative distance, using the confidence rating scale. A "nearer" square (at $94 \mathrm{in}$.) and a "farther" square (at $96 \mathrm{in}$.), both subtending equal retinal angles, were presented singly for $2 \mathrm{sec}$ in random order at several different levels of illumination (including Variac-off). S judged whether the square was in front of the line ("nearer") or behind it ("farther") and also gave a rating of his certainty.

As noted above, the purpose of these trials was to select Variac settings yielding three different levels of distance detection. In addition, these trials gave the Ss practice in judging relative distance and in using the confidence rating scale. Five of the Ss required five 1-h long sessions and a total of approximately 1000 distance judgments before the three levels of distance discrimination were obtained. One $O$ required six sessions. A final session of $1 \mathrm{~h}$ was devoted to practice judgments for size.

The experimental trials were then begun. Each $S$ made a total of 3000 size judgments and 800 distance judgments. The first experimental session was $1 \mathrm{~h}$ long; this was followed by seven 2-h sessions. In each session (except the first), $\mathbf{S}$ made 400 size judgments, with a brief 2-min rest after every 60 trials and a 10-min break after 200 trials. The 800 distance judgments were also spread equally over the eight experimental sessions. $S$ made a total of 150 size judgments for each of the $(5 \times 4=20)$ size-distance combinations. The combinations were presented randomly in blocks of 40 trials. Each combination occurred twice within a block, once with the comparison stimulus for size presented first and once with the standard stimulus viewed first. Thus in each 2-h session $\mathrm{S}$ made 20 size judgments for each of the 20 combinations. The same procedure was followed for the distance judgments. Each of the four levels of distance detection was presented randomly 10 times within a block of 40 trials. On one-half of the 10 trials at a given level the nearer stimulus was presented first; the farther stimulus was presented first during the other five trials. Stimuli for both size and distance trials were exposed for 2 sec. $S$ judged both size and distance in each session. The order of presentation of size and distance trial blocks was alternated from session to session; on one-half of the days Ss judged on a distance-size-distance sequence, with a sizedistance-size sequence for the remainder. After completing the experiment, each $\mathrm{S}$ was questioned about his strategies for detecting relative size during the experiment.

\section{RESULTS}

Figure 1 presents the results of the experiment in summary fashion. In this figure, accuracy of size judgments for the group as a whole is plotted against accuracy of distance judgments. The measure of discrimination used $\left(d_{s}\right)$ (Clarke, Bridsall, \& Tanner, 1959), is derived from detectability theory (Egan, 1958; Swets, 1964; Swets, Tanner, \& Birdsall, 1961; Weintraub \& Hake, 1962) and has the advantage of being a relatively pure measure of perceptual sensitivity uncontaminated by the criteria used by the 0 . Positive values of $d_{s}$ indicate greater than chance accuracy; negative values indicate that the Os were performing at less than chance level. In the present study negative values could occur by systematically overweighting a cue in such a manner that it would be detrimental to accurate size discrimination.

The $d_{s}$ values in Fig. 1 are mean scores, obtained by averaging $d_{s}$ scores over the six individual Ss. Individual $d_{s}$ scores, in turn, were obtained from ROC curves (Swets, 1964), based on S's partitioning of his judgments through the six confidence rating categories. These ROC curves were plotted on graph paper with normal/normal coordinates to give a straight line curve. The first point of the functions in Fig. 1 indicates accuracy of size judgments when the Variac was set at zero. The other three points represent increasing Variac settings, and thus increasing levels of distance detection.

It is obvious from Fig. 1 that there was a strong interaction between size discrimination and detectability of distance. When the farther comparison square subtended a retinal angle larger than the nearer square, increases in distance detectability led to increases in accuracy of actual size judgments. However, improvement in accuracy of distance judgments resulted in poorer performances when the farther square appeared smaller than the nearer square. A priori, it is reasonable to assume that the $d_{s}$ values for the two smaller stimuli should equal those of their larger complements when the light is off. It is clear from Fig. 1 that they do not. Verbal reports from the Os suggest that at least one reason for the differences is based on O strategy in the lights-off condition: 


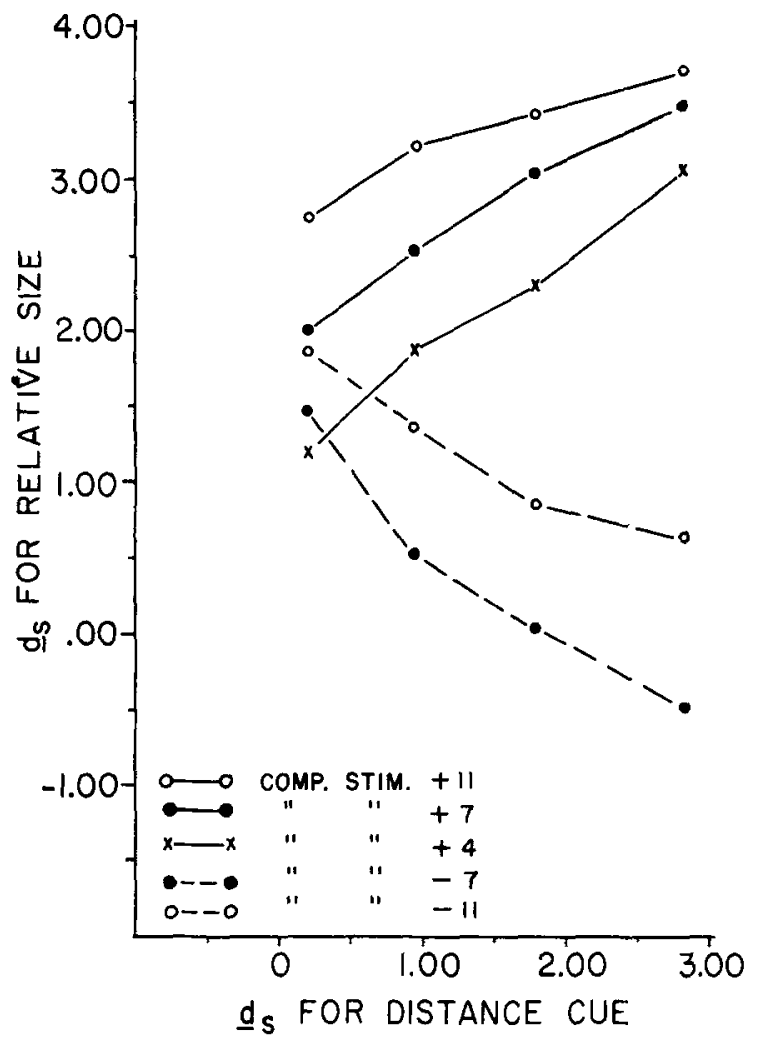

Fig. 1. Discrimination of relative size as a function of distance cue detection for $\mathrm{N}=6 \mathrm{Ss}$.

Ss reported that they used the top and sides of the viewing tunnel distal aperture as reference points to gauge relative size and found this most helpful when the stimuli were large.

All Ss showed improved accuracy with increasing distance detection when the farther square was larger than the nearer stimulus, and decreasing accuracy when the farther square was smaller. However, Table 1 indicates that the Os differed in their use of the information provided by the distance cue.
For some Ss, knowledge of relative distance has a marked effect on size judgments; for others, increases in detectability of distance led to only minor modifications in judgment of size. The Os' verbal reports were consistent with their behavior on the size judgment task. S2, for example, reported during the postexperimental inquiry that she relied heavily on her judgments of relative distance (even in the lights-off condition) in judging actual size. Conversely, S3 reported difficulty in combining retinal image and distance in making judgments of actual size, and for this reason he gave greater weight to apparent size differences.

\section{DISCUSSION}

The results clearly show the interaction of retinal image and distance discrimination in judgments of actual size. The findings are also consistent with previous research in demonstrating (1) changes in accuracy of size judgments associated with improvement in distance detectability (Holway \& Boring, 1941; Over, 1963), and (2) the overcompensation for distance when making judgments of actual size (Carlson, 1960, 1962; Epstein, 1963).

The data become more meaningful when interpreted in the context of the conditions prevailing during the experiment and in the light of the supplementary data obtained after the experimental trials were completed. The instructions to the Os did not specify the values of some variables that could have been useful to them in making their size and distance judgments. For example, neither the number nor the dimensions of the stimulus pairs were given. Nor were Ss informed about the distances, absolute or relative, of the stimuli, other than the fact that the two members would never be at equal distances from the Ss. With only this minimal information, the Os were forced to rely somewhat on previous experience and current strategies devised for the judgment situation. These $S$ factors probably enhanced the inter-O differences noted in the results.

While the evidence is only suggestive because of the small number of Ss and the possible unreliability of verbal reports, apparently an important element in determining inter-S differences in accuracy was an $O$ 's decision on the relative importance of the two cues, retinal image and distance. S1, S2, and S4 showed the greatest effect for distance, and these Ss

Table 1

Summary of $d_{s}$ Scores for the Six Observers over All Conditions (Five Squares and Four Variac Settings)

\begin{tabular}{llrrrrrrr}
\hline $\begin{array}{c}\text { Comparison } \\
\text { Square }\end{array}$ & Variaca & S1 & \multicolumn{1}{c}{ S2 } & S3 & S4 & S5 & S6 & Av \\
\hline$+11 \mathrm{~min}$ & & & & & & & & \\
\hline & Var 0 & 2.92 & 1.34 & 2.78 & 3.10 & 3.10 & 3.14 & 2.73 \\
& Var 1 & 2.42 & 2.40 & 3.31 & 3.50 & 3.60 & 3.70 & 3.16 \\
& Var 2 & 2.54 & 2.64 & 3.16 & 3.90 & 3.80 & 3.86 & 3.32 \\
+7 min & Var 3 & 3.54 & 3.28 & 3.22 & 3.96 & 3.56 & 4.00 & 3.59 \\
& Var 0 & 0.82 & 1.36 & 2.34 & 2.68 & 2.24 & 2.46 & 1.98 \\
& Var 1 & 1.34 & 1.78 & 2.64 & 3.36 & 2.72 & 3.10 & 2.49 \\
& Var 2 & 2.60 & 1.86 & 2.70 & 3.36 & 3.40 & 3.60 & 2.92 \\
$+4 \mathrm{~min}$ & Var 3 & 3.00 & 2.80 & 2.68 & 4.00 & 3.68 & 3.88 & 3.32 \\
& Var 0 & 0.56 & 1.02 & 1.00 & 1.24 & 0.96 & 2.22 & 1.17 \\
& Var 1 & 0.72 & 1.46 & 1.60 & 2.64 & 2.30 & 2.32 & 1.84 \\
& Var 2 & 1.90 & 2.02 & 1.76 & 2.70 & 1.66 & 3.06 & 2.18 \\
$-7 \mathrm{~min}$ & Var 3 & 2.36 & 3.14 & 1.92 & 3.46 & 2.60 & 3.90 & 2.90 \\
& Var 0 & 1.10 & 0.00 & 1.24 & 2.86 & 1.80 & 1.52 & 1.42 \\
& Var 1 & 1.08 & -0.54 & 0.96 & -0.30 & 1.28 & 0.54 & 0.50 \\
& Var 2 & 0.10 & -0.84 & 0.26 & -0.40 & 0.60 & 0.16 & -0.02 \\
& Var 3 & -1.04 & -1.68 & 0.36 & -1.24 & 0.40 & -0.50 & -0.62 \\
$-11 \mathrm{~min}$ & Var 0 & 1.58 & 0.24 & 2.26 & 2.50 & 2.50 & 1.92 & 1.83 \\
& Var 1 & 1.46 & -0.20 & 1.76 & 1.30 & 1.84 & 1.76 & 1.32 \\
& Var 2 & 0.46 & -1.00 & 1.48 & 0.66 & 1.80 & 1.44 & 0.81 \\
& Var 3 & 0.16 & -1.20 & 1.00 & 0.76 & 1.46 & 1.42 & 0.60 \\
\hline
\end{tabular}

$a$ Variac 0 equals Variac off; Variac 3 is the highest Variac setting. Variac Settings 1 and 2 are intermediate settings. 
also reported that they relied strongly on the distance cue, when available, in making their size judgments. The remaining three Os said they gave the greatest weight to the apparent (retinal) size of the squares.

Weighting the distance cue heavily did not mean that these Ss perceived the farther square to be more distant than did the remaining three Os. The three Ss giving greater weight to distance judged the distance between the near and far square to be, respectively, 2 in. (S1), 30 in. (S2), and 6 in. (S4); for $\mathrm{S} 3$, S5, and S6 the judgments were, respectively, 6 in., 18 in., and $38 \mathrm{in.}$ It appears, then, that assigning significance to distance did not mean that the relative distance between stimuli was perceived as being greater than the case where retinal image was given greater weight. Rather, the important factor seems to have been the general decision to use the distance cue.

In the context of this experiment, the decision to use the distance cue had differential effects on accuracy. If the farther stimulus was larger than the nearer stimulus, reliance on the distance cue enhanced accuracy; if the farther stimulus was smaller, use of the distance cue impeded performance. Questioning the Ss after the experiment suggests why this was so. Ss reported little difficulty with the size-judgment task when they perceived a square to be both phenomenologically larger and farther than the standard. In this case, they deduced (correctly) that the more distant square had to be actually larger. When distance cues were impoverished, Ss were forced to rely almost totally on perceived size differences between the two stimuli. This had the effect of increasing uncertainty in size judgments, but the Ss still performed at higher than chance levels. The most difficult judgment situation occurred when the $O$ perceived a square as both farther and smaller than the standard. In this case, $\mathrm{S}$ was faced with a difficult decision: In what way should distance and size be combined? Unlike the situation in which the comparison appeared both larger and farther, the case of a farther, phenomenologically smaller square presents an ambiguous choice, for it is possible for such a square to be either actually larger or smaller than a nearer stimulus. At this point the $O$ was forced to rely on a general strategy to help him make a decision. If he chose to weight the distance cue heavily in making his judgment, he (incorrectly) decided that the farther stimulus' phenomenological size did not correspond to its real size. If he chose to give distance little weight, his performance did not show as large a decrement in accuracy.

All Ss modified their judgments of size (correctly with the larger stimuli, incorrectly with the smaller stimuli) to some extent as the distance cue became more discriminable. That is, all the Os compensated in varying degrees for distance. It is hypothesized that this compensation had two sources. First, the demand characteristics of the experimental situation suggested that a knowledge of the relative distance of the two stimuli was useful and important in making judgments of size. Second, the Os' everyday experience led them to assume a "perspective attitude" (Carlson, 1960)-the notion that apparent size becomes smaller with increasing distance-and suggested that they should compensate for differences in distance. If a square was judged to be farther but perceived to be only slightly smaller in apparent size than a nearer object, the $O$ "overcorrected" for distance. This, apparently, is what occurred with the comparison stimulus which was 7 min of angle smaller than the standard. Greater differences in apparent size would make the $\mathrm{O}$ less likely to overcorrect for distance because of the unambiguous nature of the size difference. This is a possible explanation for the fact that the comparison stimulus $11 \mathrm{~min}$ smaller than the standard was less susceptible to overcompensation for distance.

It should be emphasized that the decrease in accuracy of size judgments which occurred with the smaller comparison stimuli resulted from a reliance on distance cues. The Ss apparently failed to consider the possibility that the distance cue might be an unreliable guide to relative size in the case where the farther stimulus was perceptually smaller than its pair-mate.

The results of the present experiment are interpreted as further confirmation of the essential importance of perceiver characteristics in the study of size-distance relationships. The failure of previous models devised to mirror these relationships, whether simplistic like the size-distance hypothesis, or elaborate like Gilinsky's (1951) model, is basically a failure to recognize the perceiver as a significant determiner of response variance.

\section{REFERENCES}

CARLSON, V. R. Overestimation in size-constancy judgments. American Journal of Psychology, 1960, 73, 199-213.

CARLSON, V. R. Size-constancy judgments and perceptual compromise. Journal of Experimental Psychology, 1962, 63, 68-73.

CLARKE, F. R., BIRDSALL, T. G., \& TANNER, W. P. Two types of ROC curves and definitions of parameters. Journal of the Acoustical Society of America, 1959, 31, 629-630.

EGAN, J. P. Recognition memory and the operating characteristic. Technical Note, Contract No. AF 19(604)-1962, 1958, Hearing and Communication Laboratory, Indiana University.

EPSTEIN, W. The known-size-apparent-distance hypothesis. American Journal of Psychology, 1961, 74, 333-346.

EPSTEIN, W. Attitudes of judgment and the size-distance invariance hypothesis. Joumal of Experimental Psychology, 1963, 66, 78-83.

EPSTEIN, W., PARK, J., \& CASEY, A. The current status of the size-distance hypothesis. Psychological Bulletin, 1961, 58, 491-514.

GILINSKY, A. S. Perceived size and distance in visual space. Psychological Review, 1951, 58, 460-482.

GILINSKY, A. S. The effect of attitude upon the perception of size. American Journal of Psychology, 1955a, 68, 173-192.

GILINSKY, A. S. The relation of perceived size to perceived distance: An analysis of Gruber's data. American Journal of Psychology, 1955b, $68,476-480$.

GOGEL, W. C., WIST, E. R., \& HARKER, G. S. A test of the invariance of the ration of perceived size to perceived distance. American Journal of Psychology, 1963, 76, 537-553.

GRUBER, $H$. E. The relation of perceived size to perceived distance. American Joumal of Psychology, 1954, 67, 411-426.

GRUBER, H. E. The size-distance paradox, a reply to Gilinsky. American Joumal of Psychology, 1956, 69, 469-476.

HASTORF, A. H., \& WAY, K. S. Apparent size with and without distance cues. American Journal of Psychology, 1952, 47, 181-188.

HOLWAY, A. H., \& BORING, E. G. Determinants of apparent visual size with distance variant. American Journal of Psychology, 1941, 54, 21-37.

JENKIN, N. Ef fects of varied distance on short-range size judgments. Journal of Experimental Psychology, 1957, 54, 327-335.

JENKIN, N., \& HYMAN, R. Attitude and distance-estimation as variable in size matching. American Journal of Psychology, 1959, 72, 68-76.

KILPATRICK, F. P., \& ITTELSON, W H. The size-distance invariance hypothesis. Psychological Review, 1953, 60, 223-231.

OVER, R. The effect of instructions on size-judgments under reduction conditions. American Journal of Psychology, 1960, 73, 599-602.

OVER, R. Size- and distance-estimates of a single stimulus under different viewing conditions. American Journal of Psychology, 1963, $76,452-457$

SMITH, W. M. Gilinsky's theory of visual size and distance. Psychological Review, 1952, 59, 239-243.

SMITH, W. M. A methodological study of size-distance perception. Journal of Psychology, 1953, 35, 143-153.

SWETS, J. A. (Ed.), Signal detection and recognition by human observers: Contemporary reading. New York: Wiley, 1964.

SWETS, J. A., TANNER, W. P., \& BIRDSALL, T. G. Decision processes in perception. Psychological Review, 1961, 68, 301-340.

WEINTRAUB, D. J., \& HAKE, H. W. Visual discrimination, an interpretation in terms of detectability theory. Journal of the Optical Society of America, 1962, 52, 1179-1184.

\section{NOTES}

1. This paper is based on a doctoral dissertation submitted to the Department of Psychology at the University of Illinois. The author is especially grateful to Charles W. Eriksen for his untiring support and guidance on this project.

2. Address: Department of Psychology, St. Norbert College, West De Pere, Wisconsin 54178.

(Accepted for publication October 11, 1968.) 